\title{
An Integrated Bi-Model Distance Regularization Method for Stem Cell Segmentation
}

\author{
Nathiya $\mathbf{R}^{1 *}$ and G Sivaradje ${ }^{2}$ \\ ${ }^{1}$ Pondicherry Engineering College, India \\ ${ }^{2}$ Electronics and Communication Engineering, Pondicherry Engineering College, India
}

Submission: June 21, 2017; Published: August 14, 2017

*Corresponding author: Nathiya R, Research Scholar, Pondicherry Engineering College, Kalapet, Puducherry -605014, India, Email: nathiya.reshmi@gmail.com

\begin{abstract}
Image processing is a lively field of research, where engineering and science disciplines cooperate together. The remarkable discovery of the prompted stem cells has been demonstrated in 2D time lapse series with a novel image processing algorithm, developed and tested for accurate segmentation and shape tracking of cells in digital images. CED filtering with a rare blend of Gaussian filtering is being applied by reducing the noise and enhancing the flow like structures with decreased execution time. The cell boundaries can be detected by Chan vese-Re-initialization model. But finding the initial curve in each time creates complexity and time consuming problem. This paper motivated to reduce the problem due to re - initialize and distance regularization. Hence integrated bi-modal distance regularized Chan-vese is proposed and implemented. Moreover this method gives better segmentation when compared with the traditional Watershed segmentation technique. The precision and recall values of the already existing Chan-vese are improved in integrated Bi-model distance regularization. In this case, two or more coupled curves evolve simultaneously to segment images with multiple objects, thereby reducing the computational cost and the level set functions. It also automatically evades the problems of vacuum and overlap. It can also represent boundaries with multiphase topologies.
\end{abstract}

Keywords: Bi-model distance regularization; Chan vese; Coherence enhance diffusion filtering; Gaussian filtering; Segmentation; Stem cell

\section{Introduction}

In stem cell specialization, the morphological features like cell size, shape, and motility [1] play an important role. Due to high demand in automatic methods, probabilistic models are generated for tracking and segmentation of phase contrast microscopy images to identify cell locations by generating cell center for each frame. Threshold based tracking and segmentation [2] are growing to monitor the proliferation and differentiation of stem cells over the lifetime. The cell properties are measured and extracted from a large number of samples for practical approaches. The topological changes in cells are automatically detected in level set [3] functions and they don't require the solution of PDE's. Initialization problems still prevails in level set functions.

The Bi model Chan-vese method affects the curve evaluation duringinitialization of curves. Multiple objects are simultaneously segmented in multiphase Chan-vese method with two or more coupled curves. Qualitative description [4] of biological processes to identify location, functional status and richness in living cells are crucial in image processing systems. Microscopy images are in demand to biologists to visualize subcellular components and process in vivo. Fluorescence microscopy [57] images have large data set with high dimensionality and give valuable source of information for contemporary biology systems. Biological analysis is indispensable to discover drugs and medicine. The image monitored with confocal microscopy yield a time lapse sequence for better analysis of segmentation. Adaptive Segmentation [6] based on active contour is used to fragment cell areas and in this method scrutinizing the data depended adoption is very challenging. Automated tracking of cell population is important to monitor the living biological specimens. The Bayesian classifiers [8] fragment the mitosis, based on posterior probability, but some mitosis region was missed due to less trailing of samples. To avoid this problem high level object classification is involved. Automated tracking of mitosis detection [9] reduce the effort of human intervention.

The quality of performance is significantly improved by reducing the undetected mitosis candidates. Quantitative evaluation [10] in HSCs (Haematopoietic stem cells) population of time lapse phase contrast microscopy images establishes lineage relationships by tracking the individual cells. Nowadays 
regimentation [11] process is popular in recent medical image processing research. The combination of segmentation and registration is called regimentation. It was first introduced by novel taxonomy algorithm and it provides consistency in image guided applications. Normally, automatic methods [12] give more powerful results than a manual process. An interactive cell segmentation based on correction propagation yields best performance to detect uncertain regions [13]. Due to the diversity morphological cell changes, creation of automatic system for segmentation is very challenging in cell analysis. Hence, under diverse conditions different samples are smeared to understand the nature of the data sets [14].

The Chan-vese model was tested out in Multiphase with major directions [15] which was mainly performed to detect the overlapping of objects. Chang-vese model prolonged with reinitialization uses a level set method for segmentation [16]. The Chan- vese model is also extended to Distance regularization [17] which does not obtain the maximum speed of segmentation.
To overcome the distance regularization problem Chan-vese Bimodal method is accomplished in re-initialization.

There are numerous proposed works in stem cell image segmentation which uses minimization of Chan-vese model in an intelligent custom [18] which combined two frameworks (FLS and GC). To overcome all the former drawbacks in this paper produces an experimental analysis of Chan-vese reinitialization model and motivated to integrate the Bimodal Chan-vese method and Distance regularization to improve the proficiency of segmentation process.

\section{Methodology}

INPUT: Time lapse series image of stem cells. In naturally slow process method, continuous projection of the frames gives an accelerated view of the process. In the above said method 8 samples are taken, with the period of 24 hours and each set is applied to the system for segmentation process followed by filtering process (Figure 1).

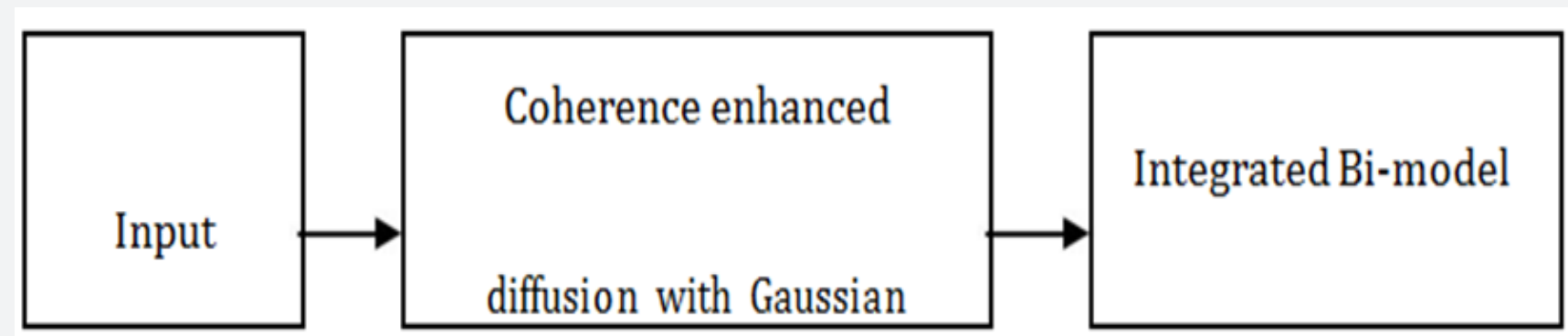

Figure 1: Flow of proposed work.

\section{Coherence-enhancing diffusion filtering}

In the paper [18], filter for enhancing coherent structures in vector-valued images have been presented based on two ideas: a generalized structure tensor for vector images, and anisotropic nonlinear diffusion filtering with a diffusion tensor. It shows that its main distinction from other color diffusion models lies in an additional integrated scale which gives a semi local average over the preferred orientation. This integration leads to significantly improved smoothing orientations, which is of importance for the enhancement of one-dimensional structures and it clarifies the role of the necessary parameters and proposed heuristics for their selection. Examples have been presented which shows that coherence-enhancing color diffusion is very robust under noise and of potential interest in various application areas. It is governed by the equation 1, 2, 3. From the paper [18].

$$
\begin{aligned}
& \partial_{t} u-d i v(D . \nabla u)=0 \text { in } I X \Omega \\
& \partial_{n}(u)=0 \text { in } I X \Omega
\end{aligned}
$$

$U(x, 0)=f(x)$

$$
\text { Where } \Omega \in R^{d} \text { is the image domain }
$$

$\mathrm{I}=|0, \mathrm{~T}|$ is the potentially in bound time interval. The essential ingredient in this equation is the diffusion tensor D of the scaled image $u$ which steers the diffusion process. It amplifies diffusion along flow-like structures, and hinders diffusion perpendicular to those patterns whose function is given by the equation 4 .

$$
\begin{aligned}
& S_{p}(u(x))=G_{p}\left(\nabla u(x) \cdot\left(\nabla u(x)^{T}\right)=\left(G_{p} *\left(\partial x_{i}(x) d x_{i} u(x)\right)\right)\right. \\
& \text { For i, j=1,2, ....d, }
\end{aligned}
$$

Here, Gp* Gaussian standard deviation of $\mathrm{p}$

However, more general averaging procedures can be used. If $\nabla u x \neq 0$ the matrix $\left(\nabla u x .(\nabla u(x))^{T}\right)$

Has rank one, the eigenvector $\nabla \mathrm{u}(\mathrm{x})$ belongs to the only non-zero eigenvalue $|\nabla u(x)| 2$. The eigen values represent the contrast in the directions of the eigen spaces. The averaging process then creates a matrix with full rank which contains valuable directional information.

This implementation is based on the semi-implicit scheme stabilized by an additive operator splitting. This scheme can be easily extended into higher dimensions and allows the use of 
longer time steps compared to explicit schemes, which results in a lower number of iterations and faster computation.

\section{Gaussian filtering}

As CED filtering is slower in its filtering process and combination of CED along with Gaussian filter will not only increase the speed of filtering but also produces an efficient output compared to the conventional filters. Gaussion filtering [19] is used to remove noise by introducing blurness in the image. It incorporates gaussion function for processing 1-dimentional image is

$$
G(x)=1 / \sqrt{2 \pi \sigma^{2} e^{x \wedge 2 / 2 \sigma \wedge 2}}
$$

Where

G(x)-Gaussion function

$\sigma$ - standard deviation of gaussion distribution.

For processing 2D- image

$$
G(x)=1 / \sqrt{2 \pi \sigma^{2} e^{x \wedge 2+y \wedge 2 / 2 \sigma \wedge 2}}
$$

\section{Where}

$\mathrm{X}$ - Distance from the origin in the horizontal axis.

$y$ - Distance from the origin in the vertical axis. $\sigma$ is the standard deviation of the distribution.

\section{Filtering involves convolution of an integral transform}

$$
I=\int \exp \left(-x^{2}\right) d x=\sqrt{\pi}
$$

Where Gaussian function is non zero as $\mathrm{x} \in[-\infty, \infty]$ and can require infinite window length. The gaussion blur to an image is same as convolving the image with a gaussion function, which has the effect of reducing the image high frequency components since the gaussion blur is a low pass filter. This filter removes gaussion noise and gives more weightage to the pixels near the edges. As the standard deviation $(\sigma)$ of the gaussion distribution increases then the degree of smoothing becomes more intensive. Gaussion fuction gives the probability distribution of data and it is a smoothing operators. It is a smoothing function and it is never equal to zero. This filter works by using 2D- Distribution as apoint spread function which is achieved by controlling the 2D gaussion function with the image. This filter is a non-uniform low pass filter which cannot preserve image brightness. It allows fast computation by giving co-efficient values nearer to zero at the edge of the mask.

\section{Segmentation}

Integrative Bi-model Distance Regularization

In order to control the smoothness of the zero level set and further avoid the occurrence of small isolated regions in the final segmentation, regularization term is added as a length penalty term $\mathrm{L}(\mathrm{C})$ which is defined related to the length of the evolving curve $C$. Let $C$ be a smooth closed planar curve $C(p):[0,1] \rightarrow \Omega$ parameterized by parameter $\mathrm{p} \in[0,1]$.The length functional can be written as

$$
L(C)=\oint d p
$$

through replacing the curve $\mathrm{C}$ by the level set function, $\mathrm{L}(\mathrm{C})$ can be reformulated as

$$
L(\phi=0)=\int_{\Omega} y \nabla(d x \phi(d / y, x)) / y=\int_{\Omega x} y \delta(\phi(d x \nabla d y)(d, x)) H^{(10)}
$$

Where, $\mathrm{H}(\mathrm{z})$ is Heaviside function and $\delta(\mathrm{z})$ Dirac delta function. The use of length penalty term implies that the evolving curve $\mathrm{C}$ which minimizes the overall energy functional should be as short as possible.It imposes a penalty on the length of the curve that separates the two phases of image, i.e., foreground and background, on which the energy functional will make a transition from one of its values, c1 (d1), to the other, c2 (d2). In many situations, the level set function will develop shocks, very sharp and or flat shape during the evolution, which in turn makes further computation highly inaccurate in numerical approximations. To avoid these problems, it is necessary to reshape the level set function to a more useful form, while keeping the zero location unchanged. A common numerical scheme is to initialize the function $\phi$.

$(\mathrm{X}, \mathrm{t}=0)$ as a signed distance function before the evolution, and then re-initialize the function $\phi(\mathrm{X}, \mathrm{t})$ to be a signed distance function periodically during the evolution, which can be written as

$$
\begin{aligned}
& \phi(X, t)=\operatorname{dist}\left(X, C_{t}\right) \quad \text { if } \mathrm{X} \text { is inside } \mathrm{C}, \\
& X \in C_{t} \quad \text { (12) } \\
& -\operatorname{dist}\left(X, C_{t}\right), \text { if } \mathrm{X} \text { is outside } \mathrm{C}_{\mathrm{t}}
\end{aligned}
$$

Where, $\operatorname{dist}(\mathrm{X}, \mathrm{Ct})$ is the shortest Euclide and instance of $\mathrm{X}$ to the points on the evolving curve $\mathrm{Ct}$ at time $\mathrm{t}$. It is crucial to keep the evolving level set function as an approximate signed distance function during the evolution, especially in the neighborhood around the zero level set.

The most straight-forward way of implementing the reinitialization operation is to extract the zero level set and then explicitly compute the distance function from it. However, this method is generally time-consuming. To overcome this difficulty, a now widely accepted method has been proposed in order to re-initialize the level set function by solving the following partial difference equation

$$
\partial \phi / \partial t=\operatorname{sign}\left(\phi_{0}\right)(1-|\nabla \phi|)
$$

Where, $\phi 0$ is the function to be re-initialized, and sign ( $\phi 0)$ is the sign function. When the steady state of above equation is reached, $\phi$ will be a distance function with the same zero level set as $\phi 0$ despite $\phi 0$ is a distance function or not. This is commonly known as the standard re-initialization procedure. Another equivalent approach is to solve the following eikonal 
equation: $|\nabla \phi|=1$

With the boundary condition on $\phi=0$ on $\left\{\phi_{0}=0\right\}$
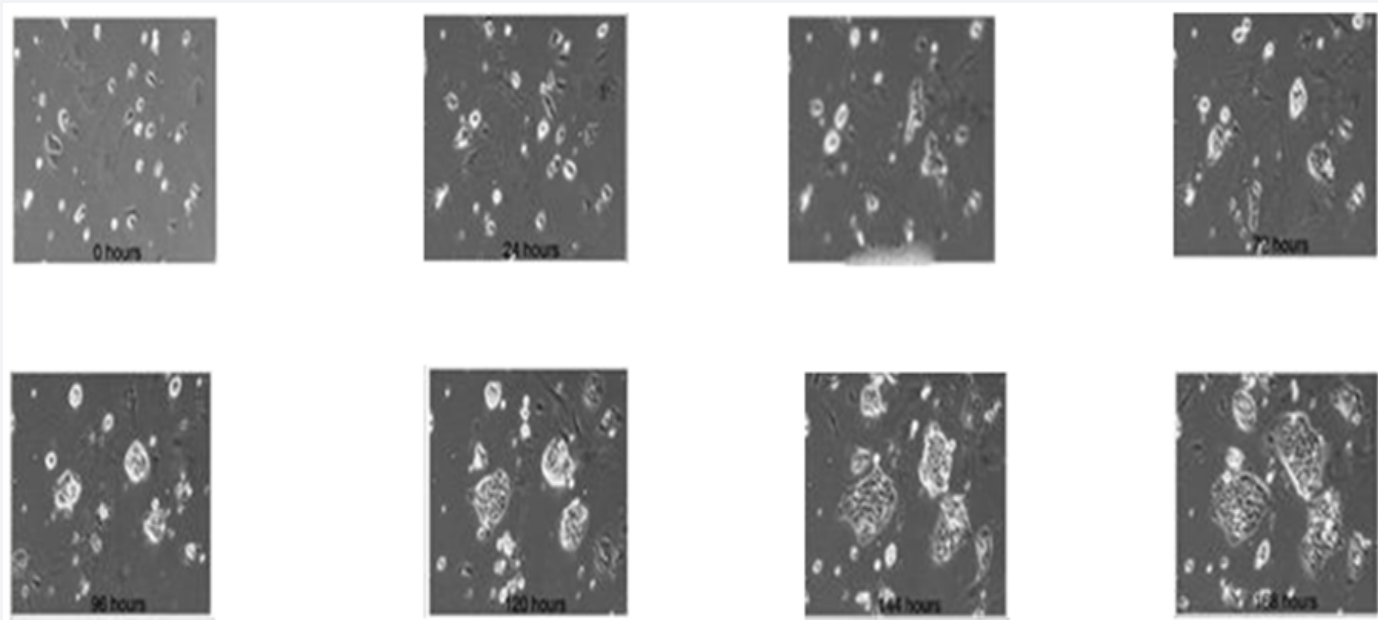

Figure 2: Time lapsed series Hematopoietic stem cell image (0 -168 hours).

\section{Experimental Analysis and Results}

\section{Outputs of combined ced and gaussian filters}

Filtering is an important pre-processing in image segmentation. To attain an efficient filtering combination of CED and Gaussian filter is used. The first 2 steps belong to CED and the final steps are trailed by Gaussian filtering process (Figure 2).

Step 1: The essential gradient is the diffusion tensor D of the scalar image which steers the diffusion process.

Step 2: Produces diffusion along flow-like structures, and hinders diffusion perpendicular to those patterns.

Step 3: Discrete approximations to the Gaussian function.
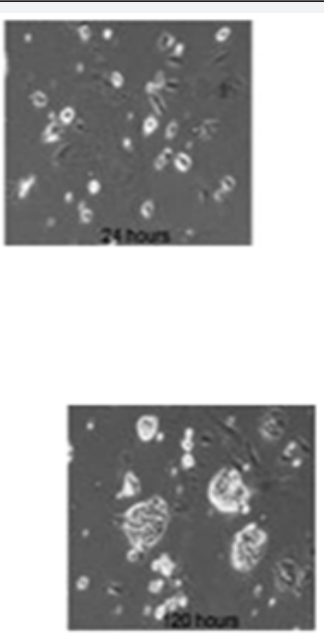

Step 4: Convolving the 2D Gaussian distribution function with the image.

Figure 2 shows that the input image of Hematopoietic stem cell image with 0-168 hours, in which 0-hour normal stem cells are presented. And after 24-hours due to mitosis proliferation process daughter cells are created. The cell growth are improved in each 24-hours.Atlast in 168-hours or in one week the total cell colony is captured.

Figure 3 shows that CED with Gaussian filtered output. In the case of CED the processing time will be more. Hence to reduce the processing time and to improve the quality of the image Gaussian is added by blurring the background pixels. So that the foreground pixels is enhanced in each set of the image.
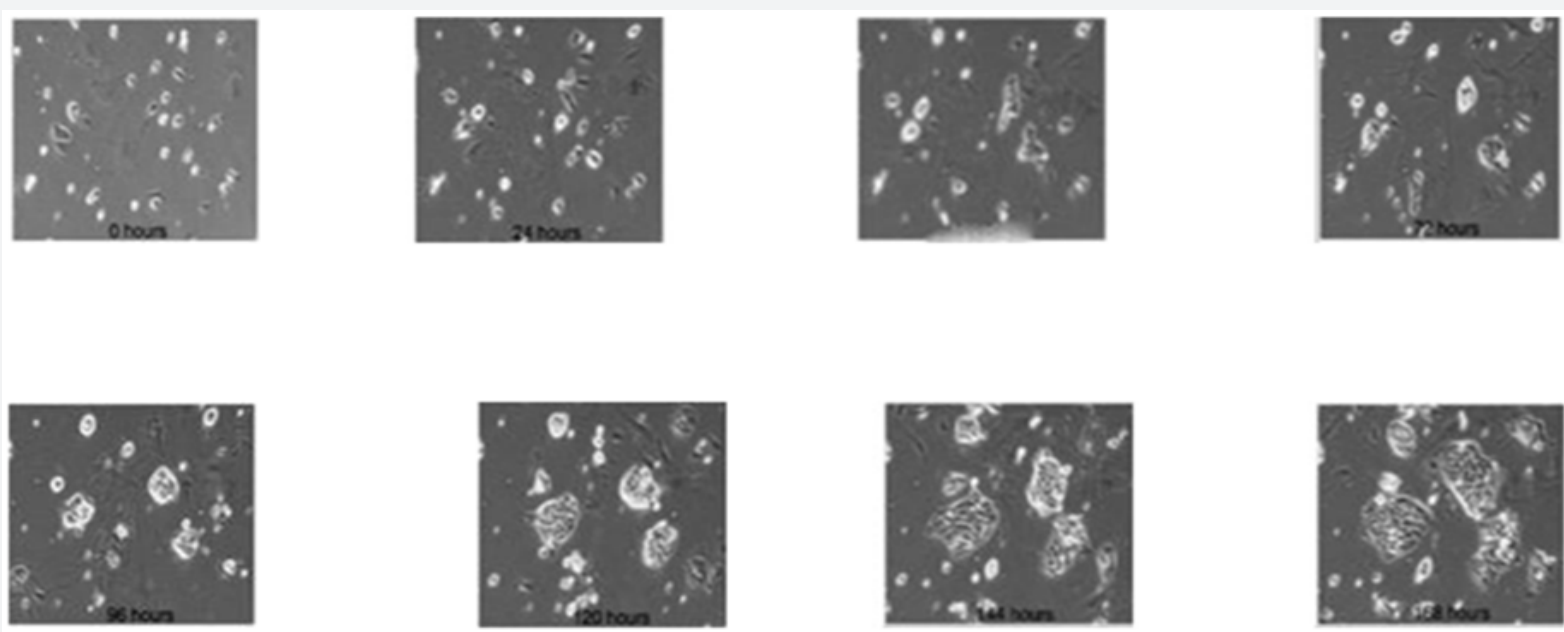

Figure 3: CED with Gaussian filtered output.

\section{Experimental results of watershed segmentation}

The watershed algorithm solves the problem of [19] oversegmentation in medical images, in this method the original image is transformed into a morphological gradient image and then the image is treated with a filter to get the reference image with less noise. Then the noise free image is segmented by using the watershed algorithm (Figure 4). 


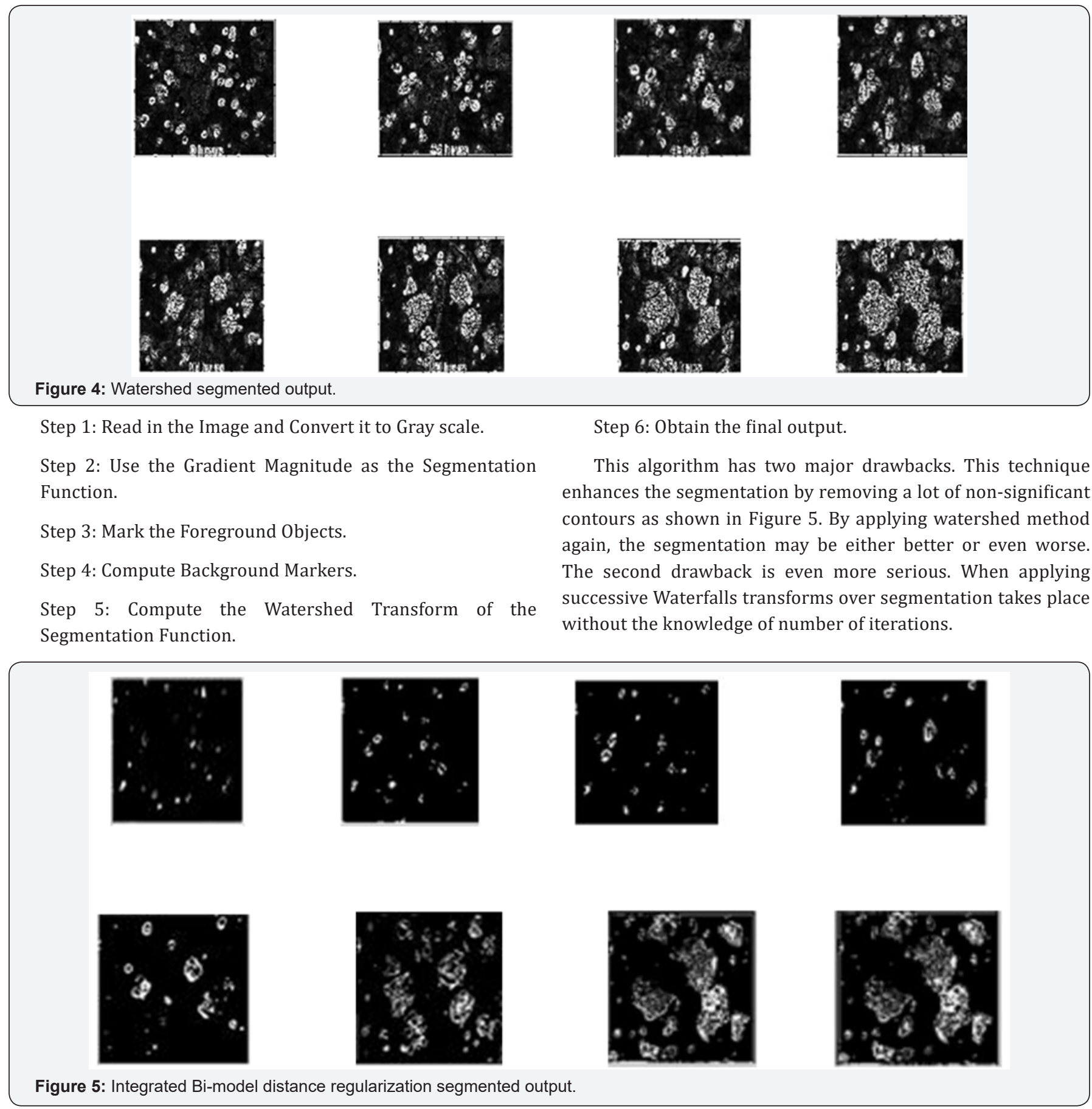

This two major problems are solved by applying bi- model integrated chan-vese algorithm. This algorithm is parametric and always ends with good segmentation of image as illustrated in the following process.

\section{Experimental results of integrated bi-modal distance regularization}

To overcome the drawback of Chan-vese algorithm due to re-initialization, Integrated bi-modal distance regularized Chan-vese algorithm is implemented. Time series images taken at the regular intervals are given as input. Figure 5 shows the simulation result of integrated Bi-modal distance regularized Chan-vese algorithm.

\section{Output parameter}

The above tabulation Table 1 gives the comparison results for the existing watershed segmentation algorithm and the proposed integrated $\mathrm{Bi}$-model distance regularization technique. These results show that the proposed technique gives better performance of $61 \%$ in terms of PSNR for 168 hours of stem cell segmentation growth. On the other hand from the above results it is evident that the watershed algorithm gives only minor 


\section{Advances in Biotechnology \& Microbiology}

performance of $40 \%$. Thus it is obvious that the integrated bimodel distance regularization technique gives effective results when compared with the existing technique.

The precision and recall values for the already prevailing watershed algorithm are $90.2 \%$ and $90.7 \%$ respectively. These values are increased by the proposed work. The obtained precision and recall from the newly proposed bi-model distance regularization technique are $98.4 \%$ and $95.2 \%$ respectively. These techniques are performed for different time durations and the matured stem cells are segmented and its PSNR values are tabulated above from 0 to 168 hours. From these results it is clear that the proposed technique gives better performance in terms of PSNR. The integrated bi-model distance regularization technique can be further improved by reducing its execution time.

Table 1: Output parameter comparison between integrated Bi-model distance regularization \& Watershed algorithm.

\begin{tabular}{|c|c|c|c|c|c|c|}
\hline Image & Precision (Bi-Model) & Precision(Watershed) & Recall (Bi-Model) & $\begin{array}{c}\text { Recall } \\
\text { (Watershed) }\end{array}$ & $\begin{array}{c}\text { PSNR(Bi- } \\
\text { Model) }\end{array}$ & $\begin{array}{c}\text { PSNR } \\
\text { (Watershed) }\end{array}$ \\
\hline 0 Hours & 0.8988 & 47.6 & 0.7783 & 33.3 & 66.83 & 48.01 \\
\hline 24 Hours & 0.9058 & 58.3 & 0.903 & 55.8 & 74.68 & 50.23 \\
\hline 48 Hours & 0.9192 & 69.7 & 0.9047 & 66 & 72.61 & 49.06 \\
\hline 72Hours & 0.9478 & 72.5 & 0.988 & 78.9 & 75.75 & 51.56 \\
\hline 96Hours & 0.9606 & 83.5 & 0.9619 & 86.2 & 74.02 & 50.22 \\
\hline 120Hours & 0.9777 & 85.5 & 0.9529 & 88.3 & 66.58 & 48.14 \\
\hline 144Hours & 0.9771 & 85.5 & 0.9554 & 90.1 & 63.61 & 43.78 \\
\hline 168Hours & 0.984 & 90.2 & 0.9518 & 90.7 & 61.1 & 40.23 \\
\hline
\end{tabular}

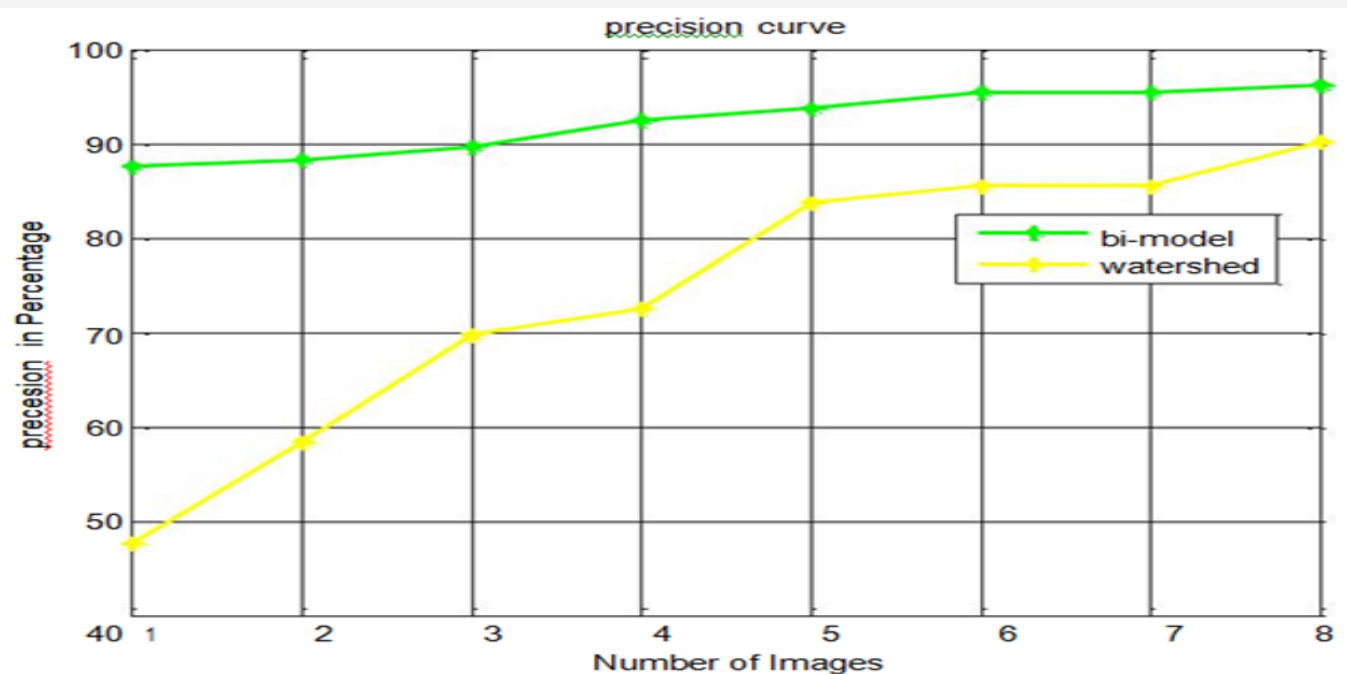

Figure 6: Precision curve for Integrated Bi-model distance regularization Chan-vese.

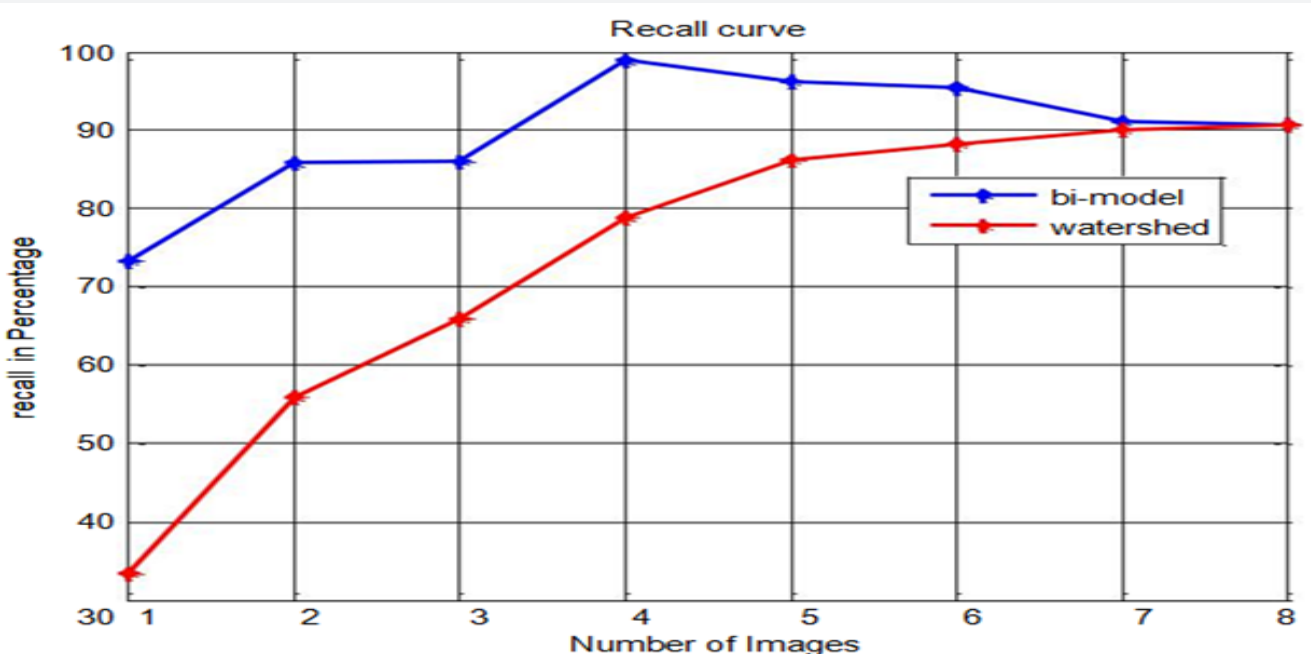

Figure 7: Recall curve for Integrated Bi-model distance regularization Chan-vese. 
The above Figure 6 gives the graphical representation of stem cell segmentation by plotting the precision values from Table 1. In this graph $\mathrm{x}$-axis indicates the number of images taken and processed with the time duration of 24 hours an the $y$-axis indicates its corresponding precision percentage values. From this graph it is clear that the proposed integrated bimodel distance regularization technique outperforms the existing watershed algorithm. This is because the watershed technique introduces over and under segmentation while processing which in turn degrades its performance thus this degradation is overcomed by implementing distance regularization in bidirectional chan-vese technique (Figure 7).

The above precision and recall curve reveals that the integrated bi-model Distance regularization method gives accurate object detection by segmenting in two directions with less level set functions when compared with the exiting watershed and chan-vese re-initialization technique which gives ineffective segmentation with intensity inhomogeneity. In Figure 6 the precision and recall curve is plotted according with true false, true negative and false negative. Hence from Table 1 the maximum segmentation accuracy of $98.4 \%$ can be achieved with the Integrated Bi-model distance regularization model.

The Chan-vese model is a piecewise constant approximation to the functional formulation of image segmentation introduced by Mumford and Shah. It has become popular in the image processing community mainly due to its ability to detect objects not necessarily defined by a gradient. Chan-vese algorithm will lead to the unsuccessful segmentation of images with intensity in-homogeneity. The sensitivity to the placement of initial contour and the extraordinary time-consumption if re-initialization step is adopted for maintaining stable curve evolution and ensuring more precise results. Therefore, Bi modal distance regularized Chan-vese algorithm is proposed. Here, the re-initialization is not directly used to keep the level set function as a signed distance function and it is more like a metric which characterizes how close a function $\phi$ is to assigned distance function. The metric plays a key role in the elimination of re-initialization. This algorithm is also capable of segmenting in two directions. Therefore, there is no possibility of missing any cells as in the case of watershed technique. The accuracy of segmentation is still increased by the usage of integrated bimodal Distance regularization algorithm.

\section{References}

1. Nezamoddin Kachouie N, Paul Fieguth, John Ramunas, Eric Jervis (2005) A Model Based Hematopoietic Stem Cell Tracker. Publications of ildverarbeitungfür die Medizin, Volume 3656, Springer, pp. 861-868.

2. Nezamoddin Kachouie N, Paul Fieguth, John Ramunas, Eric Jervis (2006) A Statistical Thresholding Method for Cell Tracking. Proceedings of IEEE International Symposium on Signal Processing and Information Technology, University of Waterloo, Canada.
3. Yongsheng Pan, Douglas Birdwell J, Seddik Djouadi M (2006) Efficient Implimentation to the Chan Vese Models without Solving PDEs. IEEE $8^{\text {th }}$ Workshop on Multimedia Signal Processing, Victoria, BC, Canada, pp. 350-353.

4. Rodrigo Fernandez Gonzalez, Arrate Munoz Barrutia, Mary Helen Barcellos Hoff, Carlos Ortiz De-Solorzano (2006) Quantitative in Vivo Microscopy: the return from the 'omics', publication of science direct, pp. 17: 501-510.

5. Cedric Vonesch, Francois Aguet, Jean Luc Vonesch, Michael Unser (2006) The coloured Revolution of Bioimaging. IEEE Signal Processing Magazine 23(3): 20-31.

6. Birgit Moller Greb, Nadine Stohr, Stefan Huttelmaier, Stefan Posch (2010) Adoptive Segmentation of Cell and Particles in Fluorescent Microscope Images. Proceedings of International Conference on Computer Vision Theory and Applications, CCIS, 229: 154-169.

7. Yung Kuan Chan, Pei Yan Pai, Chia Chi Liu, Ying Siou Wang, Ching Wu $\mathrm{Li}$, et al. (2012) Fluorescence Microscopic Image Cell Segmentation. International Journal of Future Computer and Communication vol. 1, p. 1.

8. Zhaozheng Yin, Ryoma Bise, Mei Chen, Takeo Kanade (2010) Cell Segmentation in Microscopy Images Using a Bag of Local Bayesian Classifiers. IEEE International Symposium on Biomedical Imaging, pp. $125-128$.

9. Seungil Huh, Sungeun Eom, Ryoma Bise, Zhaozheng Yin, Takeo Kanade, et al. (2011) Mitosis Detection for Stem Cell Tracking in Phase Contrast Microscopy Images.

10. Seungil, Sungeun Eom, Dai Fei Elmer Ker, Lee Weiss, Takeo Kanade, et al. (2012) Mitosis Detection of Hematopoietic Stem Cell Populations in Time Lapse Phase Contrast Microscopy Images. pp. 390 - 393.

11. Erdt M, Steger S, Sakas G (2012) Regmentation: A New View of Image Segmentation and Registration. Proceeding of Journel of Raddiation Oncology Informatics 4(1): 1-23.

12. Hang Su, Zhaozheng, Takeo Kanade, Seungil Huh (2014) Interactive Cell Segmentation Based on Correction Propagation. IEEE $11^{\text {th }}$ International Symposium on Biomedical Imaging (ISBI) Beijing, China.

13. Muhammad Jamal, Chun Lie, Christina chan, Seungik Baek, Xiaoming Liu, et al. (2014) Image Segmentation of Mesenchymal Stem Cells in Diverse Culturing Conditions. IEEE Winter Conference on Applications of Computer Vision (WACV). Steamboat Springs, CO, USA.

14. Luminita Vese A, Tony Chan F (2002) A Multiphase Level Set Framework for Image Segmentation Using the Mumford and Shah Model. International Journal of Computer Vision 50(3): 271-293.

15. Robert Crandall (2009) Image Segmentation Using the Chan-vese Algorithm. ECE 532 Project Fall.

16. Chunming Li, Chenyang XU, Changfeng GUI, Fox MD (2010) Regularized Level Set Evolution and Its Application to Image. IEEE Trans Image Process 19(12): 3243-3254.

17. Maska M, Danek O, Garasa S, Rouzaut A, Munoz Barrutia A (2013) Segmentation and Shape Tracking of Whole Fluorescent Cells based on the Chan-vese Model. IEEE Trans Med Imaging 32(6): 995-1006.

18. Chen Bao, Chen Sheng (2013) A parameterized logarithmic image processing method based on Laplacian of Gaussian filtering for lung nodules enhancement in chest radiographs. $2^{\text {nd }}$ International Symposium on Instrumentation and Measurement, Sensor Network and Automation (IMSNA), pp. 649-652.

19. Prochazka A, Vysata O, Jerhotova E (2010) Wavelet use for reduction of watershed transform over. Segmentation in Biomedical images processing, $10^{\text {th }}$ IEEE international conference on Information Technology and Applications in Biomedicine (ITAB), pp. 1-4. 
Your next submission with Juniper Publishers will reach you the below assets

- Quality Editorial service

- Swift Peer Review

- Reprints availability

- E-prints Service

- Manuscript Podcast for convenient understanding

- Global attainment for your research

- Manuscript accessibility in different formats ( Pdf, E-pub, Full Text, Audio)

- Unceasing customer service

Track the below URL for one-step submission https://juniperpublishers.com/online-submission.php 